\title{
Formation Flight and Collision Avoidance for Multiple UAVs using Concept of Elastic Weighting Factor
}

\author{
Seunghoon Kang, Hyunjin Choi and Youdan Kim* \\ School of Mechanical and Aerospace Engineering, Seoul National University, Seoul 151-742, Korea
}

\begin{abstract}
In this paper, the guidance law for formation flight and collision avoidance of multiple Unmanned Aerial Vehicle (UAV)s is proposed. To construct the physically comprehensible guidance law for formation flight, the virtual structure approach is used. To develop a guidance law for collision avoidance considering both other UAVs and unknown static obstacles, a geometric approach using information such as a relative position vector is utilized. Through the Lyapunov theorem, the stability of the proposed guidance law is proved. To combine guidance commands, the concept of the elastic weighting factor inspired by the elastic behavior of shape memory polymer, which tends to regain its original shape after deformation, is introduced. By using the concept of elastic weighting factor, multiple UAVs are able to cope actively with the situation of a collision between both UAVs and static obstacles during the formation flight. To verify the performance of the proposed method, numerical simulations are performed.
\end{abstract}

Key words: Unmmaned Aerial Vehicle, Formation Flight, Collision Avoidance, Elastic Weighting Factor

\section{Introduction}

Recently, the use of UAVs has been expanded in both military applications and civil operations [1-3]. When a UAV performs a mission such as surveillance, it is more advantageous to utilize multiple UAVs rather than a single UAV [4]. The task can be performed in a group of multiple UAVs, which can deal with the failure due to the loss of a UAV from an enemy attack or hardware/software fault. In addition, the range of operations can be extended.

To make UAVs move in formation, it is required that a proper method for formation flight which has physical reasonability be proposed and the proposed method should have a definite performance in terms of exactness of formation configuration. Formation flight of UAVs can be accomplished by several methods, which include the leader-follower approach, virtual structure approach, behavioral approach, and so on [5]. Among those methods, the virtual structure approach is more physically comprehensible compared to the others and can easily construct a formation shape.
To make UAVs cope with collision threats instantly, it is required that a proper method for collision avoidance which is simple and effective be proposed. Collision avoidance for a UAV can be accomplished by various methods such as the potential field method, geometric approach, grid based method, generic algorithm, and so on [6-7]. Among those methods, the potential field method is quite attractive in that it is easy to understand intuitively and its potential function, which is used to generate commands, can be made freely according to specific objectives. The geometric approach is also easy to understand and can be used simply.

For the operation of multiple UAVs, it is important to consider both formation flight and collision avoidance. In Ref. [8], formation flight and collision avoidance were considered separately and changed the modes according to the flight situation. In Refs. [9] and [10], formation flight and collision avoidance were considered at the same time with weighted sum of certain functions. Those works [8-10] lack effort for considering formation flight and collision avoidance simultaneously. Especially, in Refs. [9] and [10], weighting
This is an Open Access article distributed under the terms of the Creative Commons Attribution Non-Commercial License (http://creativecommons.org/licenses/by$\mathrm{nc} / 3.0 /$ which permits unrestricted non-commercial use, distribution, and reproduction in any medium, provided the original work is properly cited. 
values used in the consideration were defined as constants, which may lead to inefficiency in coping with unexpected collision threats. Also, there is no convincing explanation for the reason why the weighting values were chosen. Thus, it is strongly required to develop the proper methods for formation flight and collision avoidance regarding multiple UAVs and connect those methods with an appropriate weighting value. The value should be defined reasonably and vary with the situation continuously.

In this thesis, a physically appropriate method for formation flight based on the decentralized virtual structure approach and a simple method for collision avoidance using the geometric approach are proposed separately. Both methods are connected by a concept of elastic weighting factor that is inspired by the behavior of the material which exists in nature. Using the concept, UAVs can deal with the collision threats including the other UAVs in formation and unknown obstacles during flight simultaneously.

This paper is organized as follows. Section 2 proposes the formation flight method based on the virtual structure approach. The stability of the proposed method is analyzed using the Lyapunov Stability Theorem. Section 3 gives the collision avoidance method based on the geometric approach, and stability analysis on the proposed method is also performed using the Lyapunov Stability Theorem. Section 4 introduces the concept of elastic weighting factor for the integration of heading commands. Section 5 provides numerical simulation results in which a performance of the proposed method is verified. Finally, section 6 is devoted to the summary of the main results and the future work.

\section{Formation Flight}

A formation flight method proposed in this section is based on the following assumptions.

Assumption 2.1. There is a certain range in which UAVs can communicate.

Assumption 2.2. UAVs can obtain information which includes the position and velocity of other UAVs within the range.

\subsection{Coordinate System and Kinematics for Guidance}

All geometric information used in this study is based on a two dimensional cartesian coordinate system. Two coordinate frames are considered; the inertial coordinate frame and body fixed coordinate frame. Position information is expressed in terms of the inertial coordinate frame. Heading angle and speed of UAVs are represented in terms of body fixed frame. Let us consider a position of a UAV expressed as follows:

$$
P_{U A V}=\left[\begin{array}{ll}
x & y
\end{array}\right]_{I}^{T}
$$

Note from Eq. (1) that the position is expressed in the inertial coordinate frame only, and therefore subscript I will be omitted in the remaining sections for simplicity. To visualize the coordinate system as shown in Fig. 1, let us consider the flight situation of a single UAV with the following velocity command:

$$
\boldsymbol{V}_{c}=\left[\begin{array}{ll}
V_{c} \cos \theta_{c} & V_{c} \sin \theta_{c}
\end{array}\right]^{T}
$$

where $\theta_{c}$ and $V_{c}$ denote heading angle command and speed command of a UAV, respectively. Let us denote $P_{U A V} \in \mathbb{R}^{2}$ as the current position vector of UAV, and $\theta$ and $V$ the current heading angle and velocity, respectively. According to the given guidance commands, a future position of UAV can be obtained from the following kinematics:

$$
\begin{aligned}
& \dot{x}=V_{c} \cos \theta_{c} \\
& \dot{y}=V_{c} \sin \theta_{c}
\end{aligned}
$$

where $V_{c}$ is bounded between the minimum speed and maximum speed of a UAV as follows:

$$
V_{\min } \leq V_{c} \leq V_{\max }
$$

\subsection{Heading Command and Speed Command}

Formation flight is performed in a way that each UAV pursues its formation position that is defined through the virtual structure. To construct the virtual structure, let us define the geometric center of UAVs as follows [11]:

$$
P_{G C}=\frac{1}{n} \sum_{i=1}^{n} P_{U A V_{i}}
$$

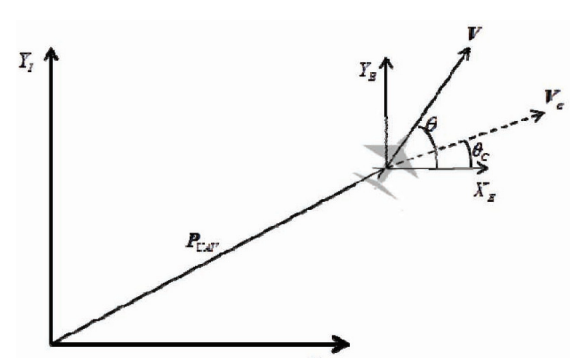

Fig. 1. Coordinate system 
where $P_{G C} \in \mathbb{R}^{2}$ and $P_{U A V_{i}} \in \mathbb{R}^{2}$ denote the position vector of the geometric center and a $i^{\text {th }} \mathrm{UAV}$, respectively, and $\mathrm{n}$ represents the total number of UAVs in group. Using $P_{G C}$, the virtual structure can be made with a proper geometric condition. The formation position of each UAV can be generated in terms of $P_{G C}$ according to the number of UAVs or a flight situation. For example, when the number of UAVs in group is 3 , that is $n=3$, a triangular shaped virtual structure and the formation position can be made as shown in Fig. 2. The whole virtual structure moves towards the goal point, and the kinematics of the formation position can also be written as:

$$
\begin{aligned}
& \dot{x}_{\text {form }}=V_{r e f} \cos \theta_{r} \\
& \dot{y}_{\text {form }}=V_{r e f} \sin \theta_{r}
\end{aligned}
$$

where $V_{\text {ref }}$ is a speed of the formation position and its value should be in the range between $V_{\min }$ and $V_{\max }$ and $\theta_{r}$ is an angle between the formation position and goal position. To consider a formation error between the UAV and the formation position, a look-ahead factor, $K$ is introduced. Considering $K$, the UAV watches the look-ahead position and generates the heading commands using the relative position between itself and the position that reflects the movement of the formation position as shown in Fig. 3.

Using the look-ahead factor $K$, the heading vector command of each UAV can be generated as follows:

$$
\begin{aligned}
\boldsymbol{r} & =\frac{\left(\boldsymbol{P}_{\text {form }}+K \dot{\boldsymbol{P}}_{\text {form }} / V_{r e f}-\boldsymbol{P}_{U A V}\right)}{\left\|\dot{P}_{\text {form }}+K \dot{\boldsymbol{P}}_{\text {form }} / V_{r e f}-\boldsymbol{P}_{U A V}\right\|} \\
& =\left[\begin{array}{ll}
\cos \theta_{c} & \sin \theta_{c}
\end{array}\right]^{T}
\end{aligned}
$$

Where $\boldsymbol{r}$ is a direction vector from the UAV to the lookahead position, and $\|\bullet\|$ represents the Euclidean norm of the vector $\boldsymbol{P}=(x, y)$ on $\mathbb{R}^{2}$.

$$
\|(x, y)\|=\sqrt{x^{2}+y^{2}}
$$

From Eq. (9), the heading command can be expressed as

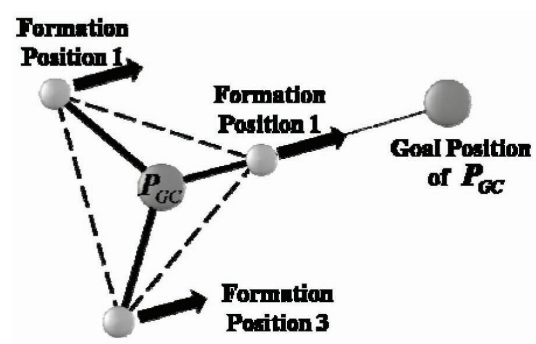

Fig. 2. Shape of virtual structure and formation positions for $n=3$ follows.

$$
\theta_{c}=\tan ^{-1}\left(\frac{r_{y}}{r_{x}}\right)
$$

where $\left(r_{x}, r_{y}\right)$ are the components of the vector $\boldsymbol{r}$.

\subsection{Stability Analysis and Speed command}

\subsubsection{Stability Analysis}

To guarantee that a position of a UAV converges to the formation position with the heading command defined in Eq. (11), a stability analysis using the Lyapunov stability theorem is performed and the detailed procedure is explained in Ref. [12].

From Ref. [12], a condition for the time derivative of the Lyapunov candidate function to be negative can be obtained as follows.

$$
\frac{V_{c} K}{\left\|\boldsymbol{P}_{\text {form }}+K \dot{\boldsymbol{P}}_{\text {form }} / V_{\text {ref }}-\boldsymbol{P}_{\text {uav }}\right\|}=V_{r e f}
$$

Eq. (12) is an important condition for deciding the lookahead factor and $V_{c}$ of a UAV, which will be analyzed in the following section.

Note from Ref. [12] that the time derivative of the Lyapunov candidate function has a negative value except the case when the position of a UAV and the formation position are coincident. This means that the value of the Lyapunov candidate function is decreasing and converges to zero asymptotically. As the Lyapunov candidate function is defined as a distance from the UAV position to formation position, it means that the distance is decreasing and become zero eventually.

\subsubsection{Look-Ahead Factor and Speed Command}

To make the heading command appropriate for the formation flight and generate a proper speed command, Eq. (12) should be considered. Two unknown variables, $K$ and $V_{c}$, have to be confirmed to generate the heading command in Eq. (9). The speed command should be within a certain bound according to the specifications of a UAV as follows:

$$
V_{\min }^{2} \leq V_{c}^{2} \leq V_{\max }^{2}
$$

Using Eq. (12), the constraint condition on the speed command can be expressed as follows:

$$
\frac{K^{2} V_{\min }^{2}}{V_{\text {ref }}^{2}} \leq\left\{x-\left(x_{\text {form }}+K \cos \theta_{r}\right)\right\}^{2}+\left\{y-\left(y_{\text {form }}+K \sin \theta_{r}\right)\right\}^{2} \leq \frac{K^{2} V_{\max }^{2}}{V_{\text {ref }}^{2}}
$$

With the properly pre-defined constant value of $K$, the flight situation of the UAV can be divided into two main cases 
using Eq. (14) as follows:

Case 1. For the case that Eq. (14) is satisfied.

: A UAV is in the stable region where the convergence to the formation position is assured.

Case 2. For the case that Eq. (14) is not satisfied.

: A UAV is outside the stable region.

The descriptions of each case are shown well in Fig. 4 .

Case 2 can be categorized again into two sub-cases.

Case. 2.1 Pursuing case

$d_{1} \leq d_{2}$

: As a projected position of a UAV is farther from the goal point than the formation position, the UAV needs to pursue the formation position.

Case. 2.2 Pursued case

$d_{1}>d_{2}$

: As a projected position of a UAV is closer from the goal point than the formation position, the UAV needs to be pursued by the formation position.

In Fig. $4, d_{1}$ is a distance between the formation position and goal position, $d_{2}$ is a distance between a projected position of the UAV which is the foot of perpendicular drawn from the UAV on the reference trajectory of the formation position and the goal position, and $d_{\text {form-ver }}$ represents a distance from the formation position to the projected position of the UAV.

Variables $K$ and $V_{c}$ corresponding to the above cases can be decided as follows:

Case 1:

$$
K=\text { constant }, \quad V_{c}=\frac{V_{\text {ref }}}{K}\left\|\boldsymbol{P}_{f o r m}+K \dot{\boldsymbol{P}}_{\text {form }} / V_{\text {ref }}-\boldsymbol{P}_{\text {uav }}\right\|
$$

Case 2:

$$
\begin{aligned}
& K=\text { constant }, \quad V_{c}=V_{\max }, \quad\left(\text { case } 2.1, d_{1}<d_{2}\right) \\
& K=d_{\text {form-ver }}+d_{v e r} \tan e, \quad V_{c}=V_{\min }, \quad\left(\text { case 2.2, } d_{1}>d_{2}\right)
\end{aligned}
$$

where $d_{v e r}, d_{\text {form-ver }}$ and $e$ are defined in Fig. 4, and $e \ll 1$.

With Eq. (9), Eq. (17) and Eq. (18), guidance commands for formation flight including the heading command and the speed command are generated. As case 2 is always turned into case 1 eventually, which means that the UAV moves towards the stable region and enters the region in the end, the convergence of the UAV to the formation position is assured in all flight situations.

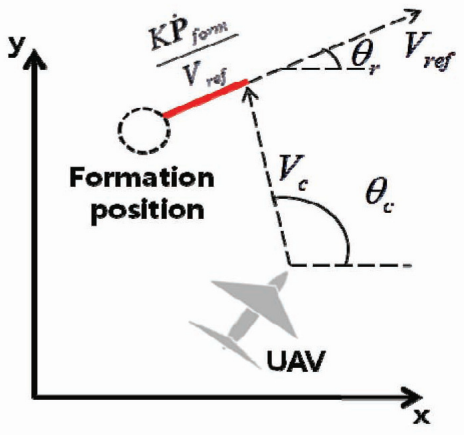

Fig. 3. Heading command defined by the position ahead of current formation position

\section{Collision Avoidance}

\subsection{Heading command}

The collision avoidance method proposed in this section is based on the following assumptions.

Assumption 3.1. Assumptions of section 2 are still valid.

Assumption 3.2. All obstacles are static.

Assumption 3.3. There exists a 'safety range' and 'sensing range' between the UAV and static obstacle.

Assumption 3.4. There exists a 'safety range' and 'sensing range' between the UAV and other UAVs.

The safety range means a minimum distance which a UAV should have for the collision avoidance of the threat. Also, the sensing range means a distance in which the UAV recognizes a threat.

The proposed method in this section deals with the procedures of generating the command for the collision avoidance of a single threat. And the method will be extended to multiple threats with a proper weighting value in section 4 .

The direction of the relative position vector can be used to increase a distance between the UAV and the threat. Another vector is also considered by defining sub-vectors which are perpendicular to the relative position vector as shown in Fig. 5. In Fig. 5, $\boldsymbol{P}_{U-T}$ is a relative position vector from the collision threat to the UAV and subscript $U$ and $T$ are the UAV and the collision threat, respectively. $\boldsymbol{P}_{s u b 1}$ and $\boldsymbol{P}_{s u b 2}$ denote sub-vectors which are perpendicular to $\boldsymbol{P}_{U-T}$ in $+90^{\circ}$ and $-90^{\circ}$, respectively. And $\boldsymbol{P}_{c m d 1}$ and $\boldsymbol{P}_{c m d 2}$ are heading vector commands which give directions for the UAV to move, which are obtained by adding $\boldsymbol{P}_{s u b 1}$ or $\boldsymbol{P}_{s u b 2}$ to $\boldsymbol{P}_{T-U}$, respectively. The collision threat can be both another UAV and an obstacle. The goal position is a formation position of the UAV when the collision threat is another UAV in the group. On the other 
hand, if the collision threat is an obstacle, the goal position is a final destination or way point of the UAV.

The heading command can be generated by using vectors which are defined in Fig. 4 as follows:

$$
\boldsymbol{P}_{c m d}= \begin{cases}\boldsymbol{P}_{c m d 1}, & \text { if } \frac{\left\|\boldsymbol{P}_{\text {goal }}-\boldsymbol{P}_{c m d 1}\right\|}{\left\|\boldsymbol{P}_{\text {goal }}-\boldsymbol{P}_{c m d 2}\right\|}<1 \\ \boldsymbol{P}_{c m d 2}, & \text { if } \frac{\left\|\boldsymbol{P}_{\text {goal }}-\boldsymbol{P}_{c m d 11}\right\|}{\left\|\boldsymbol{P}_{\text {goal }}-\boldsymbol{P}_{c m d 2}\right\|} \geq 1\end{cases}
$$

In Eq. (19), $\boldsymbol{P}_{c m d 1}$ and $\boldsymbol{P}_{c m d 2}$ can be written as follows:

$$
\boldsymbol{P}_{c m d i}=\boldsymbol{P}_{U-T}+\boldsymbol{P}_{s u b i}, \quad i=1,2
$$

In Eq. (20), i denotes the mode of the sub-vector, and decides the direction, i.e., $+90^{\circ}$ for $i=1$ and $-90^{\circ}$ for $i=2$. And $\boldsymbol{P}_{U-T}$ and $\boldsymbol{P}_{\text {subi }}$ can be written as follows:

$$
\begin{aligned}
\boldsymbol{P}_{U-T} & =\boldsymbol{P}_{U A V}-\boldsymbol{P}_{\text {Threat }}=\left[\begin{array}{ll}
x & y
\end{array}\right]^{T}-\left[\begin{array}{ll}
x_{\text {Threat }} & y_{\text {Threat }}
\end{array}\right]^{T} \\
& =\left[\begin{array}{ll}
\Delta x & \Delta y
\end{array}\right]^{T} \\
\boldsymbol{P}_{\text {subi }} & =T_{i} \cdot \boldsymbol{P}_{U-T}
\end{aligned}
$$

In Eq. (22), $T_{i}$ denotes a rotation matrix expressed as:

$$
T_{i}=\left[\begin{array}{cc}
\cos \left(-\frac{\pi}{2}(-1)^{i}\right) & -\sin \left(-\frac{\pi}{2}(-1)^{i}\right) \\
\sin \left(-\frac{\pi}{2}(-1)^{i}\right) & \cos \left(-\frac{\pi}{2}(-1)^{i}\right)
\end{array}\right]
$$

Using Eq. (21) and Eq. (23), $\boldsymbol{P}_{c m d}$ can be rewritten as follows:

$$
\boldsymbol{P}_{c m d}=\left[\Delta x+(-1)^{i} \Delta y \quad \Delta y-(-1)^{i} \Delta x\right]^{T}
$$

Using Eq. (27), the heading vector which the UAV should follow can be written as:

$$
\begin{aligned}
\boldsymbol{r}_{c} & =\frac{\boldsymbol{P}_{c m d}}{\left\|\boldsymbol{P}_{c m d}\right\|}=\left[\begin{array}{ll}
\cos \theta_{c} & \sin \theta_{c}
\end{array}\right] \\
& =\left[\begin{array}{ll}
\frac{\Delta x+(-1)^{i} \Delta y}{\sqrt{2\left(\Delta x^{2}+\Delta y^{2}\right)}} & \frac{\Delta y-(-1)^{i} \Delta x}{\sqrt{2\left(\Delta x^{2}+\Delta y^{2}\right)}}
\end{array}\right]
\end{aligned}
$$

Finally, the heading command can be generated by using Eq. (25) as:

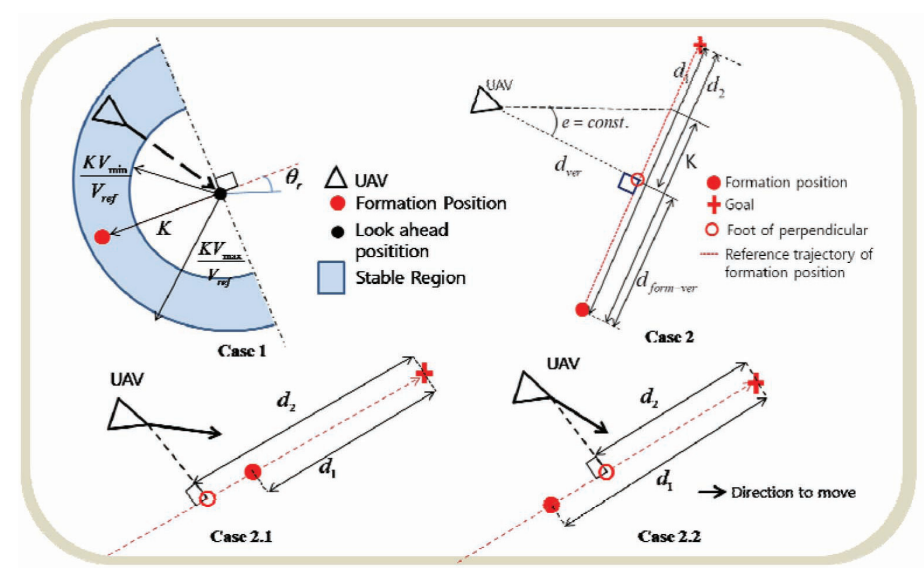

Fig. 4. Flight situations of a UAV

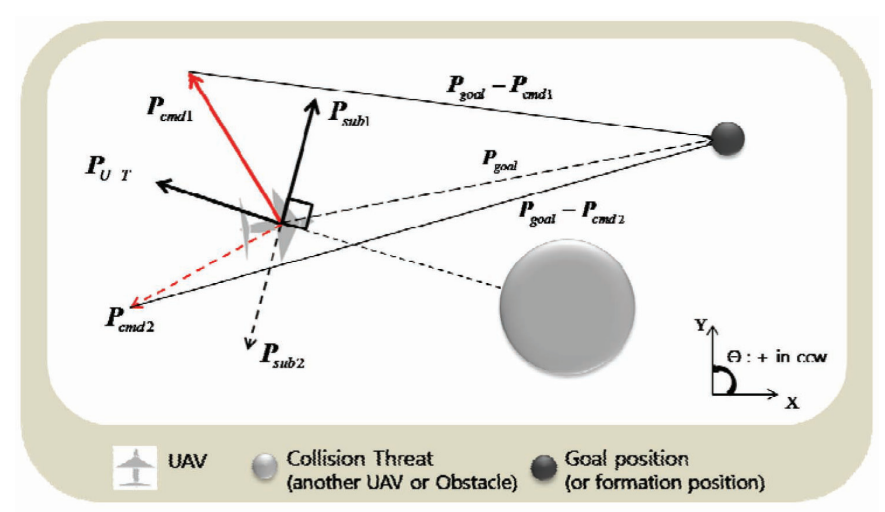

Fig. 5. Collision avoidance scheme 


$$
\theta_{c}=\tan ^{-1}\left(\frac{\boldsymbol{r}_{c, y}}{\boldsymbol{r}_{c, x}}\right)
$$

where $\boldsymbol{r}_{c, x}$ and $\boldsymbol{r}_{c, y}$ denote a x-component and a $\mathrm{y}$-component of the heading vector, $\boldsymbol{r}_{c}$, respectively.

A stability analysis for the proposed heading commands is performed using the Lyapunov stability theorem. The analysis assures that the UAV always moves in the direction away from the threat and avoids the collision threat surely whether it is another UAV or a static obstacle. Ref. [12] contains a detailed procedure of the analysis.

\section{Combined Guidance Law for Formation Flight and Collision Avoidance}

\subsection{Inspiration and Concept}

In general, the virtual structure approach deals with a fixed shape of formation. In other words, the structure is notionally treated as a rigid body. In this type of structure, the group of UAVs cannot react effectively to unexpected situations including a collision. This problem can be solved by giving the elastic property to the structure using the Elastic Weighting Factor (EWF). A main idea of the EWF is to provide an elastic property to the virtual structure as shown in Fig. 6. Using this concept, the structure of the formation is deformable during flight, and therefore UAVs can easily cope with the collision threat.

Among many materials in the real world, SMP (Shape Memory Polymer) has properties of the deformable formation structure. The SMP has the shape memory effect and the elastic behavior which can be applied to the structure. Depending on the external stimulus like temperature, the shape or length of the SMP is variable. When the external stimulus returns to the certain condition, the SMP recovers its permanent or original shape. Using this property, the formation structure can be deformed considering an effect from the collision threat and return to its original state. The formation structure can have a soft phase or a state like a rubber band for the collision avoidance as well as a hard phase or a rigid state for maintaining the formation structure.

When adapting the SMP to the formation structure, the elastic modulus of the SMP corresponds to the material property of the structure and the temperature which is an external stimulus corresponds to a distance from a UAV to the collision threat. In other words, the structure's property varies according to an interaction between the UAV and all the threats within the sensing range in which the UAV recognizes the risk of collision.

\subsection{Shape Memory Polymer Modeling}

In this section, the model of SMP is represented. The elastic weighting factor, $E$, is a value that describes the elasticity of the virtual structure and contributes to combining commands of formation flight and collision avoidance. Based on Ref. [13], $E$ can be defined mathematically as follows

Definition 4.1 (Elastic Weighting Factor, $E$ ) $E$ is a value such that

$$
E=\left\{\begin{array}{cl}
1 & \left(T<T_{s}\right) \\
\left(E_{s}-E_{g}\right) \cos \left(\frac{\pi}{2} \frac{T-T_{s}}{T_{g}-T_{s}}\right)+E_{g} & \left(T_{s} \leq T<T_{g}\right) \\
\left(E_{f}-E_{g}\right) \cos \left(\frac{\pi}{2} \frac{T-T_{f}}{T_{g}-T_{f}}\right)+E_{g} & \left(T_{g} \leq T \leq T_{f}\right) \\
0 & \left(T>T_{f}\right)
\end{array}\right.
$$

where

$$
\begin{array}{lr}
T=d_{\text {sense }}-d_{U-T}, & T_{s}=d_{\text {sense }}-d_{\text {sense }} \\
T_{f}=d_{\text {sense }}-d_{\text {safe }}, & T_{g}=\left(T_{s}+T_{f}\right) / 2 \\
E_{s}=E\left(T_{s}\right), \quad E_{g}=E\left(T_{g}\right), & E_{f}=E\left(T_{f}\right)
\end{array}
$$

Note that subscript $s$ represents the sensing range where a transition from a rigid to elastic state starts, and subscript $f$ represents the safety range where the transition terminates, subscript $g$ represents the point where an elastic property becomes predominant over a rigid property, and $T$ represents the difference between $d_{\text {sense }}$ and $d_{U-T}$.

$E$ decreases as the distance is getting closer, which means that the elastic modulus of the structure decreases. This makes the structure more flexible. On the other hand, as the distance becomes farther, the structure becomes rigid with increase in $E$.

\subsection{Combination of Heading Commands}

To adapt $E$ to the combination of heading commands for formation flight and collision avoidance, let us redefine $E$ for $i^{\text {th }} \mathrm{UAV}$ with considering all the threats.

Definition 4.2 (E for $i^{\text {th }} \mathrm{UAV}$ ) The elastic weighting factor for the $i^{\text {th }}$ UAV which is in danger of collision with several UAVs and static obstacles is defined as:

$$
E_{\text {uavi }}=\prod_{j \in \text { all }}^{p} E_{j} \prod_{k \in C A}^{q} e_{k}
$$

where the value of $p$ represents the number of all UAVs within the range in which communication is possible. The value of $q$ is the number of static obstacles detected by $i^{\text {th }} \mathrm{UAV}$, and $E_{j}$ and $e_{k}$ are portions of $E_{u a v_{-} i}$ which are related to the $j^{\text {th }} \mathrm{UAV}$ and the $k^{\text {th }}$ obstacle within the sensing range, respectively, as follows: 


$$
\begin{aligned}
& E_{j}=E\left(T_{U-U_{j}}\right)=E\left(d_{\text {sense }}-d_{U-U_{j}}\right) \\
& e_{k}=E\left(T_{U-O_{k}}\right)=E\left(d_{\text {sense }}-d_{U-O_{k}}\right)
\end{aligned}
$$

In Eq. (30), $d_{U-U_{j}}$ is a distance from the $i^{\text {th }} \mathrm{UAV}$ to the $j^{\text {th }} \mathrm{UAV}$ and $d_{U-O_{k}}$ is a distance from the $i^{t h}$ UAV to the $k^{\text {th }}$ obstacle. Using definition 2, the heading vector which the $i^{\text {th }}$ UAV should have can be written as follows:

$$
\boldsymbol{r}_{c_{-} i}=E_{\text {uav }-i} \boldsymbol{r}_{\text {form }}+\sum_{j \in a l l}^{p}\left(1-E_{j}\right) \boldsymbol{r}_{\text {CA_uav }_{-} j}+\sum_{k \in C A}^{q}\left(1-e_{k}\right) \boldsymbol{r}_{C_{-} \_ \text {obstacle } \_k}
$$

In Eq. (31), $\boldsymbol{r}_{\text {form }}$ and $\boldsymbol{r}_{C A}$ are the heading vector commands for the formation flight and the collision avoidance, respectively, i.e., Eq. (9) and Eq. (25). Using Eq. (31), the combined heading command for the $i^{\text {th }} \mathrm{UAV}$ can be written as:

$$
\theta_{c_{-} i}=\tan ^{-1}\left(\frac{\boldsymbol{r}_{c_{-} i, y}}{\boldsymbol{r}_{c_{-} i, x}}\right)
$$

where $\boldsymbol{r}_{c_{-} i, x}$ and $\boldsymbol{r}_{c_{-} i, y}$ represent the x-component and the y-component of $\boldsymbol{r}_{c_{-} i}$, respectively. Since Eq. (31) considers all the collision threats within the sensing range, the UAV can always consider the multiple collision threats during the formation flight. Especially, when UAVs fly in an unknown region, they can cope with the abrupt obstacles continuously and actively using Eq. (29).

\section{Numerical Simulation}

\subsection{Simulation Environment}

The two-dimensional point mass model of UAV is used in the simulations. To consider the time delay of UAV dynamics and autopilot, heading angle $\theta$ and speed $V$ are modeled as follows:

$$
\begin{array}{ll}
\dot{x}=V \cos \theta & \dot{y}=V \sin \theta \\
\dot{V}=\frac{\left(V_{c}-V\right)}{\tau_{V}} & \dot{\theta}=\frac{\left(\theta_{c}-\theta\right)}{\tau_{\theta}}
\end{array}
$$

where $\tau_{V}$ and $\tau_{\theta}$ represent time constants of the speed and the heading angle, respectively.

To check the performance of the proposed method, a situation of collision avoidance during the formation flight is simulated. That is, UAVs encounter unknown static obstacles during the formation flight. It is required for UAVs to avoid the collision threats by deforming the formation. After the avoidance is finished, UAVs have to recover their formation.

The parameters and initial conditions of the simulation environment are summarized in Table 1.

\subsection{Simulation Result}

Figure 7 shows the trajectories of each UAV. It can be seen that the collision avoidance during the formation flight is performed successfully. The elastic behavior of the formation structure is shown in Fig. 8. When UAVs recognize the collision threats, the formation structure varies elastically so that UAVs can avoid the threats effectively, and then recover its original formation after the avoidance. In Fig. 9, fluctuations are observed in the speed of UAVs,

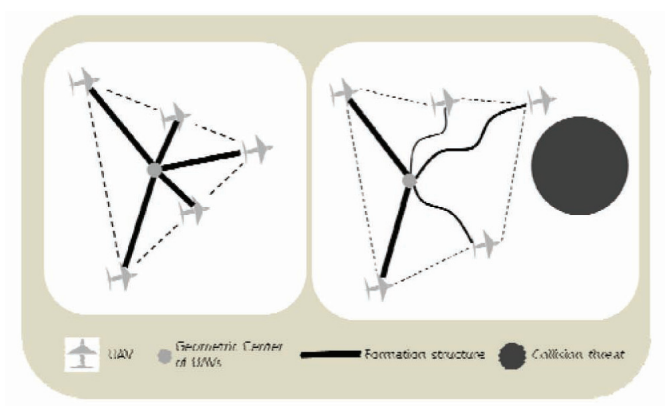

Fig. 6. Conceptual diagram of the formation structure with elasticity.

Table 1. Parameters and initial values

\begin{tabular}{|c|c|c|c|}
\hline UAV1 & $(-41.15,-0.02)^{*}$ & Heading3 & $0 \mathrm{rad}$ \\
\hline UAV2 & $(-51.54,5.98)^{*}$ & Heading4 & $0 \mathrm{rad}$ \\
\hline UAV3 & $(-61.93,11.99)^{*}$ & Heading5 & $0 \mathrm{rad}$ \\
\hline UAV4 & $(-61.93,-12.02)^{*}$ & $d_{\text {safe }}$ (UAV) & $5 \mathrm{~m}$ \\
\hline UAV5 & $(-51.54,-6.02)^{*}$ & $d_{\text {sense }}$ (UAV) & $12 \mathrm{~m}$ \\
\hline Goal of $\boldsymbol{P}_{G C}$ & $(900,0)^{*}$ & $d_{\text {safe }}($ Obstacle) & $50 \mathrm{~m}$ \\
\hline Obstacle1 & $(90,0)^{*}$ & $d_{\text {sense }}($ Obstacle) & $100 \mathrm{~m}$ \\
\hline Obstacle2 & $(170,50)^{*}$ & $V_{\max }$ & $20 \mathrm{~m} / \mathrm{s}$ \\
\hline Obstacle3 & $(275,-50)^{*}$ & $V_{\min }$ & $12 \mathrm{~m} / \mathrm{s}$ \\
\hline Obstacle4 & $(390,40)$ & Initial $V_{i}, i=1 \sim 5$ & $16 \mathrm{~m} / \mathrm{s}$ \\
\hline Obstacle5 & $(475,5)^{*}$ & $V_{\text {ref }}$ & $16 \mathrm{~m} / \mathrm{s}$ \\
\hline Heading1 & $0 \mathrm{rad}$ & $\tau_{\theta}=\tau_{V}$ & $0.2 \mathrm{sec}$ \\
\hline Heading2 & $0 \mathrm{rad}$ & Step size & 0.02 \\
\hline \multicolumn{4}{|c|}{$*$ Dimension of position $:(\mathrm{m}, \mathrm{m})$} \\
\end{tabular}


because UAVs repeatedly come in and out of the region where the stability is assured as the collision avoidance and the formation flight are considered simultaneously. Heading angles vary quite smoothly as shown in Fig. 9. In Fig. 10, it also can be seen that the concept of elastic weighting factor is used well in the presence of unknown obstacles. In addition, elastic weighting factors change continuously considering both other UAVs and unknown obstacles. In Fig. 11, all UAVs converge to their formation position after the avoidance.

To see the performance of collision avoidance with threats

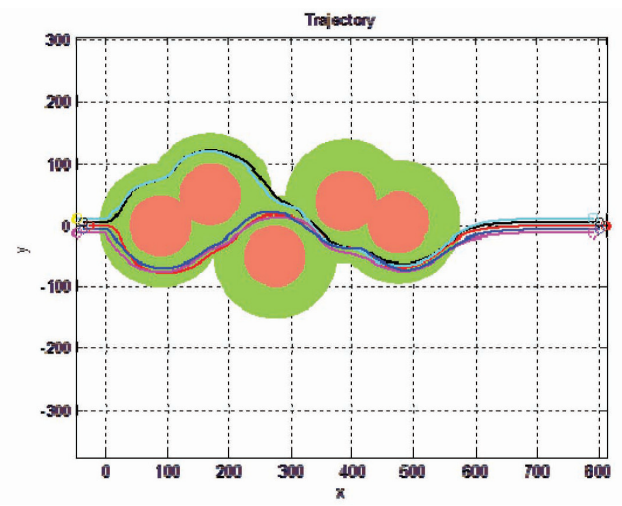

Fig. 7. Trajectories of UAVs.
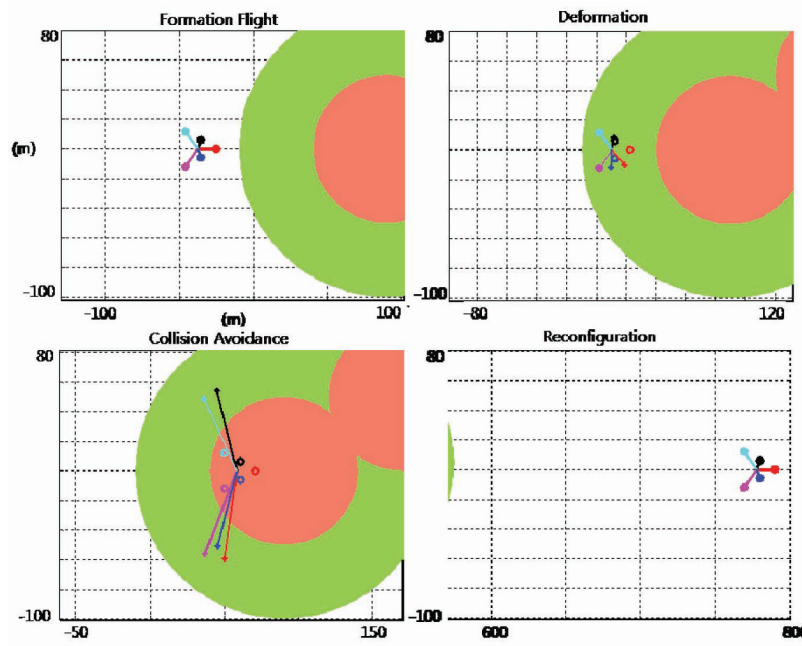

Fig. 8. Elastic behavior of the formation structure. including other UAVs and obstacles, the minimum distances among all UAVs are summarized in Table 2 and minimum distances from each UAV to the unknown obstacles are summarized in Table 3. As it can be seen in Tables 2 and 3, all UAVs maintain their distance farther than the safety range from other UAVs and obstacles. Thus, it can be said that collision avoidance during the formation flight is well performed.

\section{Conclusion}

Guidance laws for decentralized formation flight and collision avoidance are proposed. The stability of the each guidance law is proved using the Lyapunov Stability Theorem. By introducing the concept of elastic weighting factor, heading angle commands of formation flight and collision avoidance are integrated. The integrated guidance law makes UAVs perform formation flight and collision avoidance simultaneously. It is shown that UAVs can cope with the collision threats including other UAVs in the formation and unknown obstacles. The guidance command
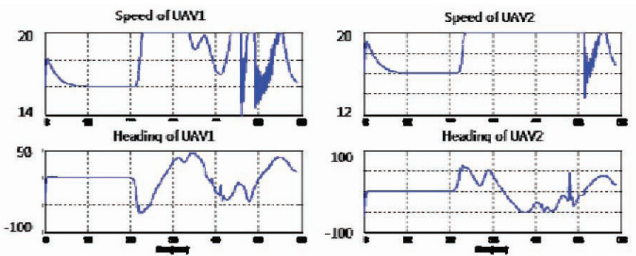

Speed of LAV 3
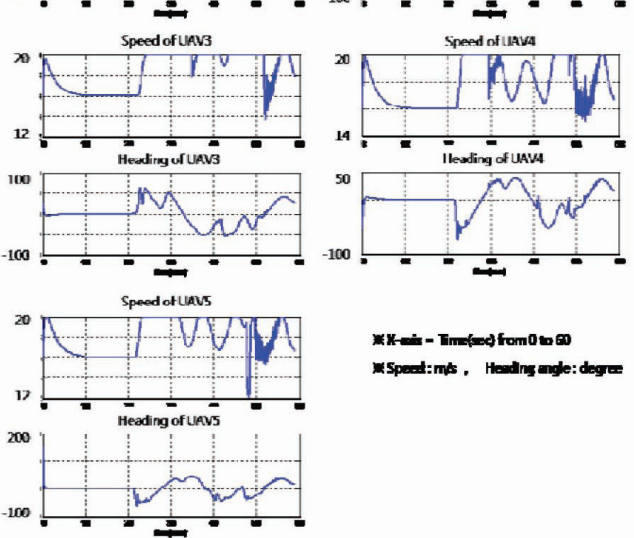

Fig. 9. Speed and heading angle histories of UAVs

Table 2. The minimum distances among all UAVs

\begin{tabular}{|c|c|c|c|c|c|}
\hline & UAV1 & UAV2 & UAV3 & UAV4 & UAV5 \\
\hline UAV1 & - & 10.3306 & 20.5457 & 13.4608 & 7.7961 \\
\hline UAV2 & 10.3306 & - & 9.1619 & 8.7658 & 7.5794 \\
\hline UAV3 & 20.5457 & 9.1619 & - & 9.0212 & 10.6476 \\
\hline UAV4 & 13.4608 & 8.7658 & 9.0212 & - & 8.0797 \\
\hline UAV5 & 7.7961 & 7.5794 & 10.6476 & 8.0797 & - \\
\hline
\end{tabular}

Dimension : (m) 
for UAVs can be generated by a simple calculation at every moment, and therefore the proposed method can be utilized for real time applications.

\section{Acknowledgement}

This work was supported by Defense Research Grant funded by Agency for Defense Development under the contract UD120013JD.

\section{References}

[1] North Atlantic Treaty Organization, Development and Operation of UAVs for Military and Civil Applications, RTO Education NOTE 9, April 2000.

[2] Z. Sarris, "Survey of UAV Applications in Civil Markets(June 2001)," the $9^{\text {th }}$ IEEE Mediterranean Conference
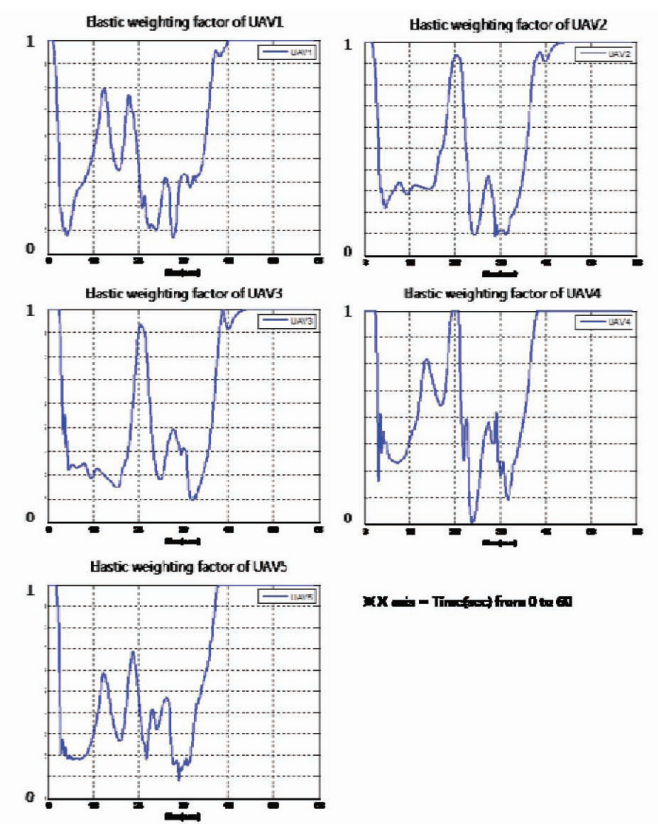

Fig. 10. Elastic weighting factor histories of UAVs on Control and Automation, Dubrovnik, Croatia , June, 2001

[3] S. Srinivasan, H. Latchman, J. Shea, T. Wong, and J. McNair, "Airborne Traffic Surveillance Systems-Video Surveillance of Highway Traffic," Proceedings of the ACM 2nd International Workshop on Video Surveillance \& Sensor Networks, New York, NY, October, 2004.

[4] C.E. Booth, Surveillance Using Multiple Unmanned Aerial Vehicles, M.S. Dissertation, Department of The Air Force Air University, Air Force Institute of Technology, Dayton, OH, March, 2009

[5] D. P. Scharf, F. Y. Hadaegh, and S. R. Ploen, "A Survey of Spacecraft Formation Flying Guidance and Control(Part II) : Control," American Control Conference, Boston, MA, July, 2004, pp. 2976-2985.

[6] B. Gardiner, W. Ahmad, T. Cooper, M. Haveard, J. Holt, and S. Biaz, "Collision Avoidance Techniques for Unmanned Aerial Vehicles Technical Report \#CSSE11-01,", Technical Report, Auburn University, Auburn, AL, August, 2011.

[7] J. Ruchti, R. Senkbeil, J. Carroll, J. Dikinson, J. Holt, and

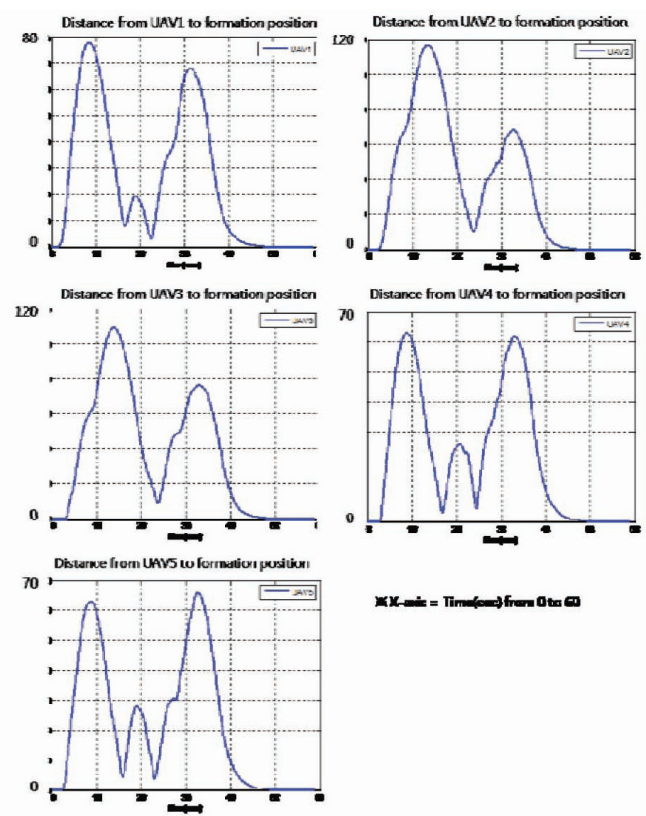

Fig. 11. Distance from each UAV to its formation position.

Table 3. The minimum distances from each UAV to unknown obstacles

\begin{tabular}{|c|c|c|c|c|c|}
\hline & UAV1 & UAV2 & UAV3 & UAV4 & UAV5 \\
\hline Obstacle1 & 68.0039 & 69.0378 & 72.0843 & 74.8391 & 65.5779 \\
\hline Obstacle2 & 79.3504 & 71.2546 & 70.6542 & 79.6127 & 70.7104 \\
\hline Obstacle3 & 67.2488 & 62.9329 & 67.7533 & 70.5871 & 72.8652 \\
\hline Obstacle4 & 69.8312 & 65.0306 & 68.3574 & 75.5162 & 67.5151 \\
\hline Obstacle5 & 65.3589 & 81.0273 & 78.2590 & 67.1168 & 67.1168 \\
\hline
\end{tabular}

Dimension : (m) 
S. Biaz, "UAV Collision Avoidance Using Artificial Potential Fields Technical Report \#CSSE11-03," Technical Report, Auburn University, Auburn, AL, July, 2011.

[8] X. Wang, V. Yadav, and S. N. Balakrishnan, "Cooperative UAV Formation Flying with Obstacle/Collision Avoidance,", IEEE Transactions on Control Systems Technology. Vol.15, Issue 4, July, 2007, pp. 672-679.

[9] C. Zhou, M. Lei, S. Zhou, and W. Zhang, "Collisionfree UAV Formation Flight Control based on Nonlinear MPC," Electronics, Communications and Control (ICECC) International Conference, Ningbo, China, September, 2011.

[10] B. Ricardo, F. Mariam, S. Joao, and G. Anouck, "Formation Control with Collision Avoidance," IEEE Conference on Decision and Control and European Control
Conference, Orlando, FL, December, 2011.

[11] J. Lee, Design of UAV Formation Flight Controller based on Formation Geometry Center Concept, M.S. Thesis, School of Mechanical and Aerospace Engineering, Seoul National University, Seoul, Korea, February, 2009.

[12] S. Kang, Formation Flight and Collision Avoidance for multiple UAVs using Concept of Elastic Weighting, M.S. Thesis, School of Mechanical and Aerospace Engineering, Seoul National University, Seoul, Korea, February, 2013.

[13] B. Zhou, Y. Lin, X. Lan, J. S. Leng, and S. H. Yoon, "A Glass Transition Model For Shape Memory Polymer And Its Composite," International Journal of Modern Physics B, Vol. 23, No. 6-7, March, 2009, pp. 1248-1253. 